\title{
Localization-induced coherent backscattering effect in wave dynamics
}

\author{
$\mathrm{H}$ Schomerus, $\mathrm{K} \mathrm{J} \mathrm{H}$ van Bemmel, and $\mathrm{C}$ W J Beenakker \\ Instutuut Lorentz. Unversitett Leiden, PO Box 9506, 2300 RA Leiden The Netherlands
}

(Received 1 September 2000, publıshed 22 January 2001)

\begin{abstract}
We investigate the statistics of single-mode delay times of waves reflected from a disordered waveguide in the presence of wave localızation The distribution of delay tımes is qualitatıvely different from the distribution in the diffusive regime, and sensitive to coherent backscattering The probability of finding small delay times is enhanced by a factor close to $\sqrt{2}$ for reflection angles near the angle of incidence This dynamic effect of coherent backscattering disappears in the diffusive regime
\end{abstract}

DOI 10 1103/PhysRevE 63026605

PACS number(s) $4225 \mathrm{Dd}, 4225 \mathrm{~Hz}, 7215 \mathrm{Rn}$

\section{INTRODUCTION}

The two most prominent interference effects arising from multiple scattering are coherent backscatterıng and wave localization [1-6] Both effects are related to the stattc intensity of a wave reflected or transmitted by a medium with randomly located scatterers Coherent backscattering is the enhancement of the reflected intensity in a nanow cone around the angle of incidence, and is a result of the systematic constructive interference in the presence of time-ieversal symmetıy [4,5] Localization arises from systematic destructive interference, and suppresses the transmitted intensity [6]

This paper presents a detailed theory of a recently discovered [7] interplay between coherent backscattering and localization in a dynamic scatteing property, the single-mode delay time of a wave reflected by a disordered waveguide The single-mode delay time is the derivative $\phi^{\prime}=d \phi / d \omega$ of the phase $\phi$ of the wave amplitude with respect to the frequency $\omega$ It is linearly ielated to the Wigner-Smith delay times of scattering theory [8-10], and is the key observable of recent experiments on multiple scattering of microwaves [11] and light waves [12] Van Tiggelen, et al [13] developed a statistical theory for the distubution of $\phi^{\prime}$ in a waveguide geometiy (where angles of incidence are discretized as modes) Although the theory was worked out manly for the case of transmission, the implications for ieflection are that the distribution $P\left(\phi^{\prime}\right)$ does not depend on whether the detected mode $n$ is the same as the incident mode $m$ or not Hence it appears that no coherent backscattening effect exists for $P\left(\phi^{\prime}\right)$

What we will demonstrate hete is that this is true only if wave localization may be disiegarded Pievious studies $[11,13]$ dealt with the diffusive regime of wavegude lengths $L$ below the localization length $\xi$ (The localization length in a waveguide geometiy is $\xi \simeq N l$, with $N$ the number of propagating modes and $l$ the mean free path) Here we consider the localized regime $L>\xi$ (assuming that also the absol ption length $\xi_{a}>\xi$ ) The distribution of reflected intensity is insensitive to the presence or absence of localization, being given in both regimes by Rayleigh's law In contıast, we find that the delay-time distribution changes matkedly as one enters the localized regime, decaying more slowly for laige $\left|\phi^{\prime}\right|$ Moreover, a coherent backscattening effect appears For $L>\xi$ the peak of $P\left(\phi^{\prime}\right)$ is higher for $n=m$ than for $n$ $\neq m$ by a factor which is close to $\sqrt{2}$, the precise factor being $\sqrt{2} \times(4096 / 1371 \pi)=135$

We also consider what happens if time-reversal symmetry 1s broken, by some magneto-optical effect The coherent backscatteing effect disappears However, even for $n \neq m$, the delay-tıme distribution for preseived time-reversal symmetry is different than for broken tıme-reversal symmetry This difference is again only present for $L>\xi$, and vanishes in the diffusive regime

The plan of this papei is as follows In Sec II we specify the notion [11] of the single-mode delay time $\phi^{\prime}$, relate 1 to the Wigner-Smith delay times, and review the results [13] for the diffusive regime, extending them to include ballistic corrections This section also contans the random-matix formulation for the localized regime, that provides the basis for our calculations, and includes a brief discussion of the conventional coherent backscattering effect in the static in tensity $I$ Section III presents the calculation of the joint distribution of $\phi^{\prime}$ and $I$ We compaie our analytical theory with numeical simulations, and give a qualitative argument for the dynamic coherent backscattering effect The role of absorption is discussed, as well as the effect of broken timereversal symmetry Deta1ls of the calculation are delegated to the Appendixes

\section{DELAY TIMES}

\section{A. Single-mode delay times}

We consider a disordered medium (mean free path $l$ ) in a waveguide geometry (length $L$ ), as depicted in $F_{1} g 1$ There ale $N \gg 1$ piopagating modes at fiequency $\omega$, given by $N$ $=\pi \mathcal{A} / \lambda^{2}$ for a waveguide with an opening of area $\mathcal{A}$ The wave velocity is $c$, and we consider a scalar wave (disregarding polatization) for simplicity In the numerical simulations we will work with a two-dimensional waveguide of width $W$, where $N=2 W / \lambda$

We study the dependence of the reflected wave amplitude

$$
I_{n m}=\sqrt{I} e^{l \phi}
$$

on the fiequency $\omega$ The indices $n$ and $m$ specify the detected and incident mode, respectively (We assume single-mode excitation and detection ) Here $I=\left|r_{n m}\right|^{2}$ is the intensity of the reflected wave in the detected mode for unit incident 


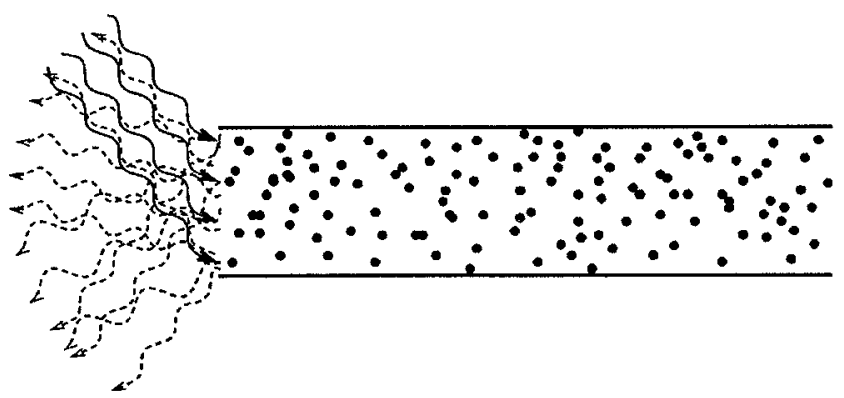

FIG. 1. Sketch of a waveguide containing a randomly scattering medium and illuminated by a monochromatic plane wave. We study the frequency dependence of the phase $\phi$ of the reflected wave amplitude in a single speckle, corresponding to a single waveguide mode. The derivative $\phi^{\prime}=d \phi / d \omega$ is the single-mode delay time.

intensity, and characterizes the static properties of the reflected wave. Dynamic information is contained in the phase derivative

$$
\phi^{\prime}=\frac{d \phi}{d \omega}
$$

which has the dimension of a time and is called the singlemode delay time $[11,13]$. The intensity $I$ and the delay time $\phi^{\prime}$ can be recovered from the product of reflection matrix elements

$$
\rho=r_{n m}\left(\omega+\frac{1}{2} \delta \omega\right) r_{n m}^{*}\left(\omega-\frac{1}{2} \delta \omega\right)
$$

evaluated at two nearby frequencies $\omega \pm \frac{1}{2} \delta \omega$. To leading order in the frequency difference $\delta \omega$ one has

$$
\rho=I\left(1+i \delta \omega \phi^{\prime}\right) \Rightarrow I=\lim _{\delta \omega \rightarrow 0} \operatorname{Re} \rho, \quad \phi^{\prime}=\lim _{\delta \omega \rightarrow 0} \frac{\operatorname{Im} \rho}{\delta \omega l}
$$

We seek the joint distribution function $P\left(I, \phi^{\prime}\right)$ in an ensemble of different realizations of disorder. We distinguish between the diffusive regime where $L$ is small compared to the localization length $\xi \simeq N l$, and the localized regime where $L \geq \xi$. Localization also requires that the absorption length $\xi_{a} \geqslant \xi$. We will contrast the case of excitation and detection in two distinct modes $n \neq m$ with the equal-mode case $n=m$. Although we mainly focus on the optically more relevant case of preserved time-reversal symmetry, we will also discuss the case of broken time-reversal symmetry for comparison. These two cases are indicated by the indexes $\beta=1$ and 2 , respectively.

\section{B. Relation to Wigner-Smith delay times}

In the localized regime $\left(\xi \ll L, \xi_{a}\right)$ we can relate the single-mode delay time $\phi^{\prime}$ to the Wigner-Smith [8-10] delay times $\tau_{l}$, with $i=1, \ldots, N$. The $\tau_{l}$ 's are defined for a unitary reflection matrix $r$ (composed of the elements $r_{n m}$ ); hence they require the absence of transmission and of $a b-$ sorption. One then has

$$
\begin{aligned}
& -i r^{\dagger} \frac{d r}{d \omega}=U^{\dagger} \operatorname{diag}\left(\tau_{1}, \ldots, \tau_{N}\right) U, \\
& -i r^{*} \frac{d r^{T}}{d \omega}=V^{\dagger} \operatorname{diag}\left(\tau_{1}, \ldots, \tau_{N}\right) V,
\end{aligned}
$$

with $U$ and $V$ unitary matrices of eigenvectors. In the presence of time-reversal symmetry $r$ is a symmetric matrix; hence $V=U$ in this case.

For small $\delta \omega$ we can expand

$$
r\left(\omega \pm \frac{1}{2} \delta \omega\right)=V^{T} U \pm \frac{1}{2} i \delta \omega V^{T} \operatorname{diag}\left(\tau_{1}, \ldots, \tau_{N}\right) U .
$$

Inserting this into Eq. (3) and comparing with Eq. (4) yields the relations

$$
\phi^{\prime}=\operatorname{Re} \frac{A_{1}}{A_{0}}, \quad I=\left|A_{0}\right|^{2}, \quad A_{k}=\sum_{t} \tau_{t}^{k} u_{t} v_{\imath}
$$

We have abbreviated $u_{i}=U_{t m}$ and $v_{l}=V_{t n}$. In the special case $n=m$, the coefficients $u_{\imath}$ and $v_{l}$ are identical in the presence of time-reversal symmetry.

The distribution of the Wigner-Smith delay times in the localized regime was determined recently [14]. In terms of the rates $\mu_{t}=1 / \tau_{t}$ it has the form of the Laguerre ensemble of random-matrix theory,

$$
P\left(\left\{\mu_{t}\right\}\right) \propto \prod_{l<j}\left|\mu_{t}-\mu_{j}\right|^{\beta} \prod_{k} \Theta\left(\mu_{k}\right) e^{-\gamma(\beta N+2-\beta) \mu_{k}},
$$

where the step function $\Theta(x)=1$ for $x>0$ and 0 for $x<0$. The parameter $\gamma$ is defined by

$$
\gamma=\alpha l / c
$$

with the coefficient $\alpha=\pi^{2} / 4$ or $8 / 3$ for two- or threedimensional scattering, respectively. Equation (8) extends the $N=1$ result of Refs. $[15-17]$ to any $N$.

The matrices $U$ and $V$ in Eq. (6) are uniformly distributed in the unitary group. They are independent for $\beta=2$, while $U=V$ for $\beta=1$. In the large- $N$ limit the matrix elements become independent Gaussian random numbers with vanishing mean and variance $1 / N$. Hence

$$
\left\langle u_{l}\right\rangle=\left\langle v_{l}\right\rangle=0, \quad\left\langle\left|u_{l}\right|^{2}\right\rangle=\left\langle\left|v_{l}\right|^{2}\right\rangle=N^{-1},
$$

with $u_{\imath}=v_{1}$ for $n=m$ and $\beta=1$. Corrections to this Gaussian approximation are of order $1 / N$.

\section{Diffusion theory}

The joint probability distribution $P\left(I, \phi^{\prime}\right)$ in the diffusive regime $l \ll L \ll \xi$ was derived in Refs. [11,13],

$$
\begin{aligned}
P_{\text {diff }}\left(I, \phi^{\prime}\right)= & \Theta(I)\left(I / \pi \bar{I}^{3}\right)^{1 / 2} e^{-I / I}\left(Q \bar{\phi}^{\prime 2}\right)^{-1 / 2} \\
& \times \exp \left(-\frac{I}{\bar{I}} \frac{\left(\phi^{\prime}-\bar{\phi}^{\prime}\right)^{2}}{Q \bar{\phi}^{\prime 2}}\right)
\end{aligned}
$$


with constants $\bar{I}, \bar{\phi}^{\prime}$, and $Q$ It has the same form for transmission and reflection, the only difference being the dependence of the constants on the system parameters Here we focus on the case of reflection, because we are concerned with coherent backscattering

From the joint distribution function [Eq (11)], for the intensity one obtains the Rayleigh distribution

$$
P_{\mathrm{d} \text { fff }}(I)=\frac{1}{\bar{I}} \exp (-I / \bar{I})
$$

Hence $\bar{I}$ is the mean detected intensity per mode It is given by $[18]$

$$
\bar{I}=\frac{1}{N}\left(1+\delta_{\beta 1} \delta_{n m}\right),
$$

assuming unit incident intensity The factor of 2 enhancement in the case $n=m$ is the static coherent backscattering effect mentioned in Sec I, which exists only in the presence of time-reversal symmetry $(\beta=1)$ Equations (12) and (13) remain valid in the localized regime, since they are determined by scattering on the scale of the mean free path Hence $L \gg l$ is sufficient for static coherent backscattering, and it does not matter whether $L$ is small or large compared to $\xi$

By integrating ove1 $I$ in Eq (11) one arrives at the distr1bution of single-mode delay times $[11,13]$,

$$
P_{\mathrm{dlff}}\left(\phi^{\prime}\right)=\frac{Q}{2 \bar{\phi}^{\prime}}\left[Q+\left(\phi^{\prime} / \bar{\phi}^{\prime}-1\right)^{2}\right]^{-3 / 2}
$$

Hence $\bar{\phi}^{\prime}$ is the mean delay time, while $\sqrt{Q}$ sets the relative width of the distribution These constants are determined by the correlator $[11,13]$

$$
\begin{aligned}
C_{12} & =\frac{\left\langle r_{n m}(\omega+\delta \omega) r_{n m}^{*}(\omega)\right\rangle}{\left\langle r_{n m}(\omega) r_{n m}^{*}(\omega)\right\rangle} \\
& =1+\imath \bar{\phi}^{\prime} \delta \omega-\frac{1}{2} \bar{\phi}^{\prime 2}(Q+1)(\delta \omega)^{2}
\end{aligned}
$$

Diffusion theory gives

$$
\bar{\phi}^{\prime}=2 \gamma s / 3, \quad Q=2 s / 5
$$

Here $\gamma$ is given by Eq (9) We have defined

$$
s=\alpha^{\prime} L / l,
$$

where the numerical coefficient $\alpha^{\prime}=2 / \pi, 3 / 4$ for two- and three-dimensional scatterıng (The corresponding iesult for $Q$ given in Ref [13] is inconect)

Diffusion theory predicts that the distiıbution of delay times [Eq (14)], as well as the values of the constants $\bar{\phi}^{\prime}$ and $Q$, do not depend on the choice $n=m$ or $n \neq m$ (and also not on whether time ieversal symmetiy is pieserved or not) Hence there is no dynamic effect of coherent backscatteing in the diffusive legime

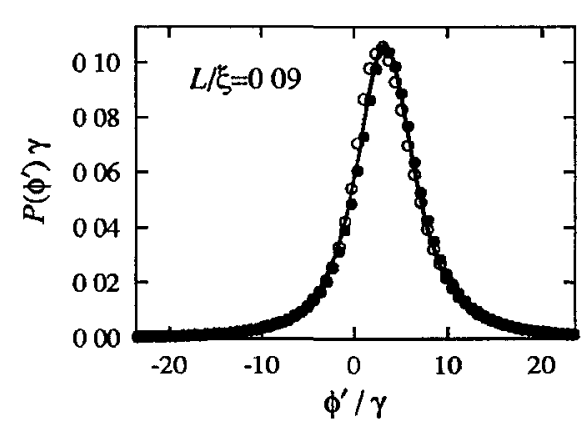

FIG 2 Distribution of the single-mode delay time $\phi^{\prime}$ in the diffusive regime The iesult of numerical simulation (data points) with $N=50$ propagating modes is compared to the prediction [Eq (14)] of diffusion theory (solid curve) There is no difference between the case $n=m$ of equal-mode excitation and detection (open circles) and the case $n \neq m$ of excitation and detection in distinct modes (full circles)

\section{Ballistuc corrections}

The expressions for the constants $\bar{I}, \bar{\phi}^{\prime}$, and $Q$ given above are valid up to corrections of order $l / L$ Heie we give more accurate formulas that account for these ballistic corrections (We need these to compare with numencal simulations ) We determine the ballistic corrections for $Q$ and $\bar{\phi}^{\prime}$ by relating the dynamic problem to a static problem with absorption (This relationship only works for the mean It cannot be used to obtain the distribution [19]) The mean total ieflectivity

$$
\bar{a}=1+x-\sqrt{2 x+x^{2}} \operatorname{coth}\left[s \sqrt{2 x+x^{2}}+\operatorname{arcosh}(1+x)\right]
$$

for absorption $\alpha^{\prime} x$ per mean free path was evaluated in Ref [20] [Heie $\alpha^{\prime}$ is the same constant as in the definition of $s$, see $\mathrm{Eq}(17)]$ We identify $C_{12}=\bar{a}(x) / \bar{a}(0)$ by analytical contınuation to an imaginaty absorption rate $x=-\imath \delta \omega \gamma$ Expanding in $x$ to second ordel, we find

$$
\bar{\phi}^{\prime}=\gamma \frac{s(3+2 s)}{3(1+s)}, \quad Q=\frac{8 s^{3}+28 s^{2}+30 s+15}{5(2 s+3)^{2}}
$$

\section{E Numerical simulation}

The validity of diffusion theory was tested in Refs [1113] by compaison with expeiıments in transmission In Fig 2 we show an alternative test in ieflection, by comparison with a numetical simulation of scattering of a scalar wave by a two-dimensional iandom medium (We assume time 1eversal symmetry) The ieflection matrices,$\left(\omega \pm \frac{1}{2} \delta \omega\right)$ are computed by applying the method of recursive Gieen functions [21] to the Helmholtz equation on a square lattıce (lattice constant $a$ ) The width $W=100 a$ and the fiequency $\omega$ $=14 \mathrm{c} / a$ are chosen such that there are $N=50$ propagating modes The mean fiee path $l=140 a$ is found fiom the formula [22] to $\prime^{\prime}=N s(1+s)^{-1}$ for the reflection probability The conesponding localization length $\xi=N L / s=1100 a$ The parameter $\gamma=463 \mathrm{a} / \mathrm{c}$ is found fiom Eq (19) by equat- 
ing $\left\langle\phi^{\prime}\right\rangle=\bar{\phi}^{\prime}$ (This value of $\gamma$ is somewhat larger than the value $\gamma=\pi^{2} l / 4 c=345 a / c$ expected for two dimensional scattering, as a consequence of the anisotiopic dispersion relation on a square lattice) We will use the same set of parameters later in this paper in the inteipretation of the results in the localized regime Our numerical results confirm that in the diffusive regime the distribution of delay times $\phi^{\prime}$ does not distinguish between excitation and detection in distinct modes ( $n \neq m$, full curcles) and identical modes ( $n=m$, open c1rcles)

\section{DYNAMIC COHERENT BACKSCATTERING EFFECT}

\section{A. Distinct-mode excitation and detection}

We now calculate the joint probability distribution function $P\left(I, \phi^{\prime}\right)$ of intensity $I$ and single-mode delay time $\phi^{\prime}$ in the localized regime, for the typical case $n \neq m$ of excitation and detection in two distinct modes We assume a preserved tıme-reversal symmetry ( $\beta=1$ ), leaving the case of broken time-1eveisal symmetry for the end of this section

It is convenient to work momentarily with the werghted delay time $W=\phi^{\prime} I$ and to iccover $P\left(I, \phi^{\prime}\right)$ from $P(I, W)$ at the end The characteristic function

$$
\chi(p, q)=\left\langle e^{-\imath p l-\imath q W}\right\rangle
$$

is the Fourier transform of $P(I, W)$ The average $\langle>$ is over the vectors $\mathbf{u}$ and $\mathbf{v}$ and over the set of eigenvalues $\left\{\tau_{t}\right\}$ The average over one of the vectors, say $\mathbf{v}$, is easily carried out, because it is a Gaussian integiation The result is a determinant

$$
\begin{gathered}
\chi(p, q)=\left\langle\operatorname{det}(1+\imath H / N)^{-1}\right\rangle, \\
H=p \mathbf{u}^{*} \mathbf{u}^{T}+\frac{1}{2} q\left(\overline{\mathbf{u}}^{*} \mathbf{u}^{T}+\mathbf{u}^{*} \overline{\mathbf{u}}^{T}\right)
\end{gathered}
$$

The Hermitian matıx $H$ is a sum of dyadic products of the vectors $\mathbf{u}$ and $\overline{\mathbf{u}}$, with $\bar{u}_{l}=u_{t} \tau_{l}$, and hence has only two non-vanishing eigenvalues $\lambda_{+}$and $\lambda_{-}$Some straightfo1ward lineaı algebra gives

$$
\lambda_{ \pm}=\frac{1}{2}\left(q B_{1}+p \pm \sqrt{2 p q B_{1}+q^{2} B_{2}+p^{2}}\right),
$$

where we have defined the spectral moments

$$
B_{k}=\sum_{l}\left|u_{l}\right|^{2} \tau_{l}^{k}
$$

The resulting determinant is

$$
\operatorname{det}(1+H / N)^{-1}=\left(1+\lambda_{+} / N\right)^{-1}\left(1+\lambda_{-} / N\right)^{-1},
$$

hence

$$
\chi(p, q)=\left\langle\left[1+\frac{l p}{N}+\frac{l q}{N} B_{1}+\frac{q^{2}}{4 N^{2}}\left(B_{2}-B_{1}^{2}\right)\right]^{-1}\right\rangle
$$

An inverse Four1er transform, followed by a change of var1ables from $I, W$ to $I, \phi^{\prime}$, gives

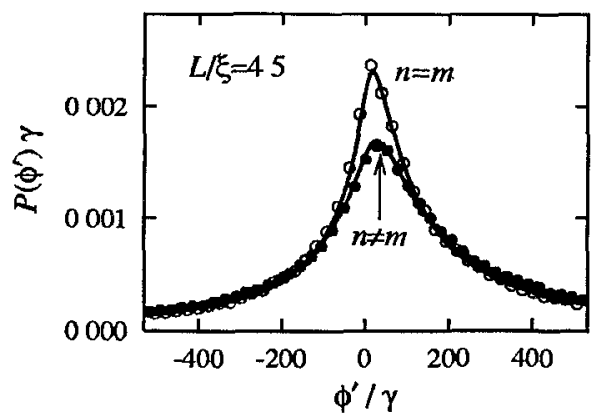

FIG 3 Distribution of the single-mode delay time $\phi^{\prime}$ in the localized regime The results of numerical simulations with $N$ $=50$ propagatıng modes (open curcles for $n=m$, full circles for $n$ $\neq m$ ) are compared to the analytical predictions The curve for dif ferent incident and detected modes $n \neq m$ is obtained from Eqs (27) and (28) The curve for $n=m$ is calculated from Eqs (29) and (30) The same value for $\gamma$ is used as in the diffusive regime (Fig 2)

$$
\begin{aligned}
P\left(I, \phi^{\prime}\right)= & \Theta(I)\left(N^{3} I / \pi\right)^{1 / 2} e^{-N I} \\
& \times\left(\left(B_{2}-B_{1}^{2}\right)^{-1 / 2} \exp \left(-N I \frac{\left(\phi^{\prime}-B_{1}\right)^{2}}{B_{2}-B_{1}^{2}}\right)\right)
\end{aligned}
$$

The average is over the spectral moments $B_{1}$ and $B_{2}$, which depend on the $u_{\imath}$ 's and $\tau_{l}$ 's via Eq (23)

The calculation of the joint distribution $P\left(B_{1}, B_{2}\right)$ is presented in Appendix A The result is

$$
\begin{aligned}
P\left(B_{1}, B_{2}\right)= & \Theta\left(B_{1}\right) \Theta\left(B_{2}\right) \exp \left(-\frac{N B_{1}^{2}}{B_{2}}\right) \\
& \times\left[\frac{B_{1}^{2} \gamma N^{3}}{B_{2}^{4}}\left(B_{2}+\gamma N^{2} B_{1}\right) \exp \left(-\frac{2 \gamma N}{B_{1}}\right)\right. \\
& \left.-\frac{\gamma^{3} N^{5}}{4 B_{2}^{5}}\left(2 B_{2}^{2}-4 B_{1}^{2} B_{2} N+B_{1}^{4} N^{2}\right) \mathrm{E}_{1}\left(-\frac{2 \gamma N}{B_{1}}\right)\right],
\end{aligned}
$$

where $\operatorname{Er}_{1}(x)$ is the exponential-1nteg1al function The distr1bution $P\left(I, \phi^{\prime}\right)$ follows fiom Eq (26) by integrating ovet $B_{1}$ and $B_{2}$ with weight given by Eq (27)

Inespective of the distribution of $B_{1}$ and $B_{2}$, fiom $\mathrm{Eq}$ (26) we recover the Rayleigh law $[\mathrm{Eq}$ (12)] for the intensity $I$ The distibution $P\left(\phi^{\prime}\right)=\int_{0}^{\infty} d I P\left(I, \phi^{\prime}\right)$ of the single-mode delay time takes the form

$$
P\left(\phi^{\prime}\right)=\int_{0}^{\infty} d B_{1} \int_{0}^{\infty} d B_{2} \frac{P\left(B_{1}, B_{2}\right)\left(B_{2}-B_{1}^{2}\right)}{2\left(B_{2}+\phi^{\prime 2}-2 B_{1} \phi^{\prime}\right)^{3 / 2}}
$$

In Fig 3 this distibution is compated with the result of a numerical simulation of a random medium as in Sec II E, but now in the localized regime The same value for $\gamma$ was used as in Fig 2, making this comparison a parameter-tree test of the theory (Note that $\gamma$ alone determines the complete distribution function in the localized regime, in contrast to 
the diffusive case where two parameters are required) The numerical data agree very well with the analytical prediction

\section{B. Equal-mode excitation and detection}

We now turn to the case $n=m$ of equal-mode excitation and detection, still assuming that time-1eversal symmetiy is preserved Since $u_{l}=v_{t}$, we now have

$$
\phi^{\prime}=\operatorname{Re} \frac{C_{1}}{C_{0}}, \quad I=\left|C_{0}\right|^{2}, \quad C_{k}=\sum_{l} \tau_{l}^{k} u_{t}^{2}
$$

The joint distribution function $P\left(C_{0}, C_{1}\right)$ of these complex numbers can be calculated in the same way as $P\left(B_{1}, B_{2}\right)$ In Appendix $\mathrm{C}$ we obtain

$$
\begin{aligned}
P\left(C_{0}, C_{1}\right) \propto & \exp \left(-N\left|C_{0}\right|^{2} / 2\right) \int_{0}^{\infty} d s s^{2} e^{-s} \\
& \times\left(1+\frac{\left|C_{1}\right|^{2} s^{2}}{\gamma^{2} N^{2}}-\frac{2 s}{\gamma N} \operatorname{Re} C_{0} C_{1}^{*}\right)^{-5 / 2}
\end{aligned}
$$

The corresponding distribution function $P\left(\phi^{\prime}\right)$ is also plotted in Fig 3, and compared with the results of the numerical simulation Good agreement is obtained, without any free parameter

\section{Comparison of both situations}

Comparing the two curves in Fig 3, we find a stuking difference between distinct-mode and equal-mode excitation and detection The distribution for $n=m$ displays an enhanced probability of small delay times In the vicinity of the peak, both distributions become very similai when the delay times for $n \neq m$ are divided by a scale factor of about $\sqrt{2}$ In the limit $N \rightarrow \infty$ (see Sec IIID), the maximal value $P\left(\phi_{\text {peak }}^{\prime}\right)=\sqrt{2 / \pi N^{3} \gamma^{2}}$ for $n=m$ is larger than the maximum of $P\left(\phi^{\prime}\right)$ for $n \neq m$ by a factor

$$
\frac{P_{n=m}\left(\phi_{\text {peak }}^{\prime}\right)}{P_{n \neq m}\left(\phi_{\text {pedk }}^{\prime}\right)}=\sqrt{2} \times \frac{4096}{1371 \pi}=135
$$

Correspondingly, the probability to find very laige delay times is reduced for $n=m$ This is reflected by the asymptotic behaviol

$$
P\left(\phi^{\prime}\right)-\frac{\gamma N^{3 / 2}}{\phi^{\prime 2}} \begin{cases}(2 \pi)^{-1 / 2} & \text { for } n=m \\ \sqrt{\pi / 4} \text { for } n \neq m\end{cases}
$$

The enhanced probability of small delay times for $n=m$ is the dynamic coherent backscatteing effect mentioned in Sec I The effect requires localization, and is not observed in the diffusive regime

\section{Limit $N \rightarrow \infty$}

The results piesented so fat assume $N \gg 1$, but retain finite- $N$ collections of order $N^{-1 / 2}$ (Only terms of order $1 / N$ and higher are neglected ) It turns out that the asymmetry of

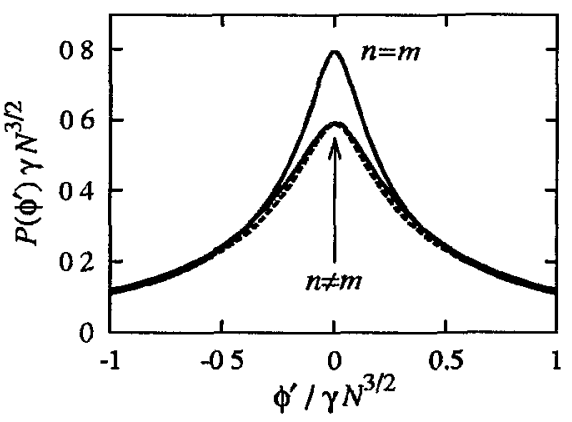

FIG 4 Distribution of the single-mode delay time $\phi^{\prime}$ in the localized regime for preserved time-reversal symmetry, in the limit $N \rightarrow \infty$ In this limit $P\left(\phi^{\prime}\right)$ becomes symmetric for positive and negative values of $\phi^{\prime}$ Compared are the result for $n \neq m$ [Eqs (33) and (A16)] and $n=m$ [Eqs (29), (34), and (35)] The distribution for $n=m$ falls on top of the distribution for $n \neq m$ when $\phi^{\prime}$ is rescaled by a factor 135 (dashed curve, almost indistınguishable from the solid curve for $n \neq m$ )

$P\left(\phi^{\prime}\right)$ for positive and negative values of $\phi^{\prime}$ is an effect of order $N^{-1 / 2}$ The asymmetry is hence captured fathfully by our calculation We now consider how the asymmetry eventually disappears in the limit $N \rightarrow \infty$

For distinct modes $n \neq m$, the spectral moments scale as $B_{1} \sim \gamma N$ and $B_{2} \sim \gamma^{2} N^{3}$ With $\phi^{\prime} \sim \gamma N^{3 / 2}$, one finds that $B_{1}$ can be omitted to order $N^{-1 / 2}$ in $\mathrm{Eq}$ (28) One obtains the symmetıic distribution

$$
P\left(\phi^{\prime}\right)=\int_{0}^{\infty} d B_{2} \frac{P\left(B_{2}\right) B_{2}}{2\left(B_{2}+\phi^{\prime 2}\right)^{3 / 2}}
$$

plotted in $\mathrm{F}_{1 \mathrm{~g}} 4$

For identical modes $n=m$, obseive that the quantities $C_{0}$ and $C_{1}$ become mutually independent in the large- $N$ limit The cross-term $(\gamma N)^{-1} \operatorname{Re} C_{0} C_{1}^{*}$ in Eq (30) is of relative order $N^{-1 / 2}$ because $C_{0} \sim N^{-1 / 2}$ and $C_{1} \sim \gamma N$ Hence, to ordel $N^{-1 / 2}$, the distribution factorizes, $P\left(C_{0}, C_{1}\right)$ $=P\left(C_{0}\right) P\left(C_{1}\right)$ The distribution of $C_{0}$ is a Gaussian,

$$
P\left(C_{0}\right)=\frac{N}{2 \pi} \exp \left(-N\left|C_{0}\right|^{2} / 2\right)
$$

as a consequence of the central-limit theorem, and

$$
P\left(C_{1}\right) \propto \int_{0}^{\infty} d s s^{2} e^{-s}\left(1+\frac{\left|C_{1}\right|^{2} s^{2}}{\gamma^{2} N^{2}}\right)^{-5 / 2}
$$

The 1esulting distribution of $\phi^{\prime}=\operatorname{Re}\left(C_{1} / C_{0}\right)$ is also plotted in Fig 4

The dynamic coherent backscattenng effect peisists in the limit $N \rightarrow \infty$, it is therefore not due to finite- $N$ corrections The peak heights differ by the factor given in Eq (31) 


\section{E. Interpretation in terms of large fluctuations}

In order to explain the coherent backscattering enhancement of the peak of $P\left(\phi^{\prime}\right)$ in more qualitative terms, we compare $\mathrm{Eq}$ (29) for $n=m$ with the corresponding relation [Eq (7)] for $n \neq m$

The factorization of the joint distribution function $P\left(C_{0}, C_{1}\right)$ discussed in $\mathrm{Sec}$ III D can be seen as a consequence of the high density of anomalously large WignerSmith delay times $\tau_{i}$ in the Laguerre ensemble [Eq (8)] The distribution of the laigest time $\tau_{\max }=\max _{l} \tau_{l}$ follows from the distribution of the smallest eigenvalue in the Laguerre ensemble, calculated by Edelman [23] It is given by

$$
P\left(\tau_{\max }\right)=\frac{\gamma N^{2}}{\tau_{\max }^{2}} \exp \left(-\gamma N^{2} / \tau_{\max }\right)
$$

As a consequence, the spectral moment $C_{1}$ is dominated by a small number of contributions $u_{t}^{2} \tau_{t}$ (often enough by a single one, say with index $l=1$ ), while $C_{0}$ can be safely approximated by the sum over all remaining indices $\imath$ (say, $\imath \neq 1$ ) The same argument applies also to the spectral moments $A_{k}$ which determine the delay-tıme statistics for $n \neq m$, hence the distribution function $P\left(A_{0}, A_{1}\right)$ factorizes as well

The quantities $A_{0}$ and $C_{0}$ have a Gaussian distribution for large $N$, because of the central-limit theorem, with $P\left(C_{0}\right)$ given by $\mathrm{Eq}(34)$ and

$$
P\left(A_{0}\right)=\frac{N}{\pi} \exp \left(-N\left|A_{0}\right|^{2}\right)
$$

It then becomes clear that the main contribution to the enhancement $[\mathrm{Eq}(31)]$ of the peak height, namely, the factor of $\sqrt{2}$, has the same origin as the factor of 2 enhancement of the mean intensity $\bar{I}$ More precisely, the relation $P\left(A_{0}=x\right)$ $=2 P\left(C_{0}=\sqrt{2} x\right)$ leads to a rescaling of $P(I)$ for $n=m$ by a factor of $1 / 2$ and to a 1escaling of $P\left(\phi^{\prime}\right)$ by a factor of $\sqrt{2}$ The remaining factor of $4096 / 1371 \pi=095$ comes fiom the difference in the distributions $P\left(A_{1}\right)$ and $P\left(C_{1}\right)$ It turns out that the distubution

$$
\begin{aligned}
P\left(A_{1}\right)= & \int_{0}^{\infty} d s \frac{1}{4 \pi \gamma N} \frac{s^{2}}{\left(4+\left|A_{1} / \gamma N\right|^{2} s^{2}\right)^{3}} \\
& \times\left[e^{-s}\left(64+32 s+12 s^{2}+s^{3}\right)-3 s^{2} \operatorname{El}(-s)\right]
\end{aligned}
$$

(derived in Appendix D) is very similar to $P\left(C_{1}\right)$ given in Eq (35), hence the remaining factor is close to unity

The laige $\tau_{l}$ 's are related to the penetiation of the wave deep into the localized tegions and are eliminated in the diffusive regime $L \leq \xi$ In Sec IIIF we compare the localized and diffusive regimes in mote detail

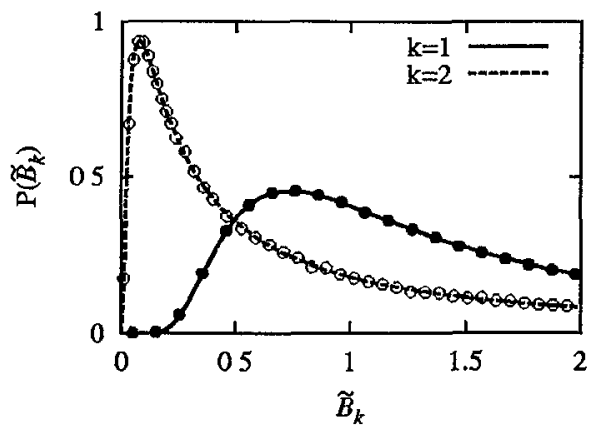

FIG 5 Distributions of $\widetilde{B}_{1}=B_{1} / \gamma N$ and $\widetilde{B}_{2}=B_{2} / \gamma^{2} N^{3}$ The analytıc prediction from $\mathrm{Eq}$ (27) [for exphicit formulas see Eqs (A15) and (A16)] is compared to the result of a numerical simulation of a Laguerre ensemble with $N=50$

\section{F. Localized vs diffusive regime}

Comparison of Eqs (11) and (26) shows that the two joint distributions of $I$ and $\phi^{\prime}$ would be identical if statistical fluctuations in the spectral moments $B_{1}$ and $B_{2}$ could be ignored The correspondences are

$$
B_{1} \leftrightarrow \bar{\phi}^{\prime}, \quad B_{2}-B_{1}^{2} \leftrightarrow Q \bar{\phi}^{\prime 2}
$$

However, the distribution $P\left(B_{1}, B_{2}\right)$ is very broad (see Fig 5 ), so that fluctuations cannot be ignoied The most probable values ate

$$
B_{1}^{\text {typical }} \simeq \gamma N, \quad B_{2}^{\text {typical }} \simeq \gamma^{2} N^{3},
$$

but the mean values $\left\langle B_{1}\right\rangle,\left\langle B_{2}\right\rangle$ diverge-demonstıatıng the presence of large fluctuations In the diffusive regime $L \leqslant \xi$ the spectial moments $B_{1}$ and $B_{2}$ can be replaced by their ensemble averages, and the diffusion theory $[11,13]$ is iecoveied (The same applies if the absoiption length $\xi_{a} \leqslant \xi$ )

The large fluctuations in $B_{1}$ and $B_{2}$ directly affect the statistical propeities of the delay time $\phi^{\prime}$ We compare the distiıbution $[\mathrm{Eq}(28)]$ in the localized regime (F1g 3) with the 1esult $[\mathrm{Eq}$ (14)] of diffusion theory (F1g 2) In the localized legime the value $\phi_{\text {pe } \mathrm{k}}^{\prime} \simeq B_{1}^{\text {typical }}$ at the center of the peak of $P\left(\phi^{\prime}\right)$ is much smaller than the width of the peak $\Delta \phi^{\prime}$ $\simeq\left(B_{2}^{\text {typical }}\right)^{1 / 2} \simeq \phi_{\text {pe } \mathrm{k}}^{\prime}(\xi / l)^{1 / 2}$ This also holds in the diffusive regime, where $\phi_{\mathrm{pe} \mathrm{lK}}^{\prime}=\bar{\phi}^{\prime}$ and $\Delta \phi^{\prime} \simeq \phi_{\mathrm{pe} \mathrm{l \textrm {k }}}^{\prime}(L / l)^{1 / 2}$ However, the mean $\left\langle\phi^{\prime}\right\rangle=\left\langle B_{1}\right\rangle$ diverges for $P$, but is finte (equal to $\left.\bar{\phi}^{\prime}\right)$ for $P_{\text {diff }}$ For large $B_{2}$ one has, asymptotically, $P\left(B_{2}\right)$ $-\frac{1}{4} N \gamma^{3 / 2} \sqrt{\pi} B_{2}^{-3 / 2}$ As a consequence, in the tarls $P\left(\phi^{\prime}\right)$ falls off only quadiatically [see Eq (32)], while in the diffusive regime $P_{\text {diff }}\left(\phi^{\prime}\right) \sim \frac{1}{2} Q \bar{\phi}^{\prime 2}\left|\phi^{\prime}\right|^{-3}$ falls off with an inverse thitd powel

\section{G. Role of absorption}

Although absoiption causes the same exponential decay of the tiansmitted intensity as localization, this decay is of a quite different, namely, an incoherent, nature The stiong fiuctuations in the localized regime disappear as soon as the absoiption length $\xi_{a}$ diops below the localization length $\xi$, because long paths which penetiate into the localized regions 

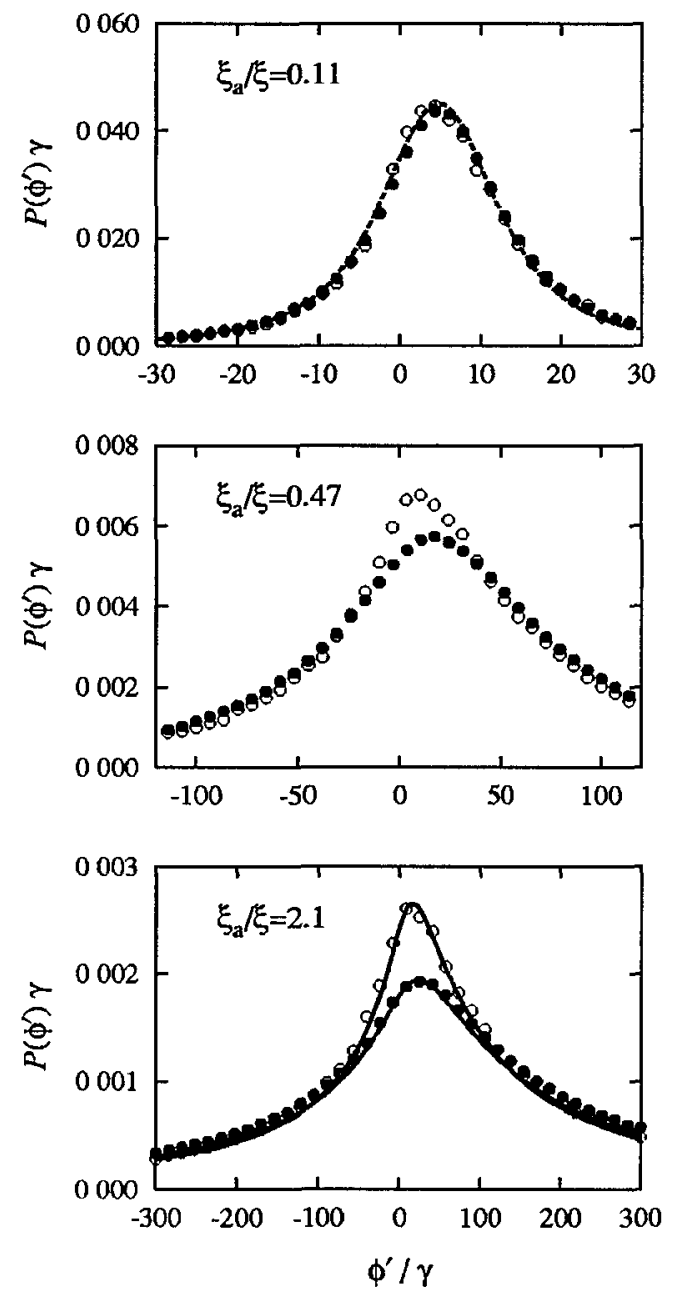

FIG 6 Single-mode delay-time distribution $P\left(\phi^{\prime}\right)$ in the presence of absorption The data points are the result of a numerical simulation of a waveguide with length $L=45 \xi$ Open circles are for equal-mode excitation and detection $n=m$, and full circles for the case of distinct modes $n \neq m$ In the upper panel (with $\xi_{a}<\xi$ ), the data are compared to the prediction [Eq (14)] of diffusion theory In the lower panel we compare with the predictions [Eqs (27)(30)] of random-matrix theory

are suppressed by absorption In this situation one should expect that the results of diffusion theory are again valid even for $L \geqslant \xi$ This expectation is confirmed by our numerical simulations (We do not know how to incorporate absorption effects into our analytical theory)

In Fig 6 we plot the delay-time distribution for two values of the absorption length $\xi_{a}<\xi$ and one value $\xi_{a}>\xi$, both for equal-mode and distinct-mode excitation and detection The length of the waveguide is $L=41 \xi$ The result for strong absoiption with $\xi_{a}=011 \xi$ is very similar to Fig 2 Irrespective of the choice of the detection mode, the data can be fitted to prediction (14) of diffusion theory The plot for $\xi_{a}=047 \xi$ shows that the dynamic coherent backscattering effect slowly sets in when the absorption length becomes comparable to the localization length The data also deviate fiom the prediction of diffusion theory The full factor $[\mathrm{Eq}$ (31)] between the peak heights quickly develops as soon as

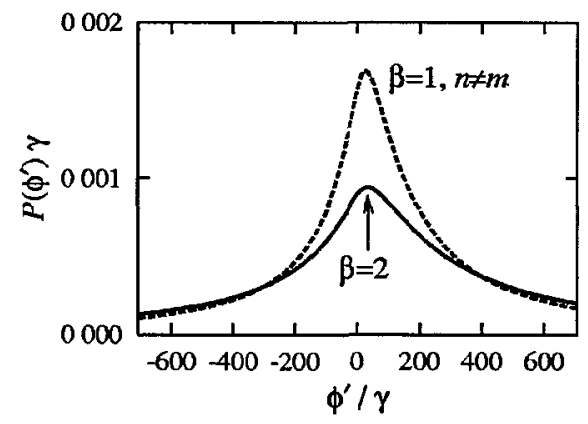

FIG 7 Comparison of the single-mode delay-tıme distributions for preserved and broken time-reversal symmetry The number of propagating modes is $N=50$ The curves are calculated from $\mathrm{Eq}$ (28), with $P\left(B_{1}, B_{2}\right)$ given by $\mathrm{Eq}(27)(\beta=1)$ or $\mathrm{Eq}(41)(\beta$ $=2$ )

$\xi_{a}$ exceeds $\xi$, as can be seen fiom the data for $\xi_{a}=21 \xi$ Moreover, these data can already be fitted to the predictions of random-matrix theory, with $\gamma \approx 532 \mathrm{a} / \mathrm{c}$ (The value $\gamma$ $=463 \mathrm{a} / \mathrm{c}$ of Sec II E is leached when absorption is further reduced )

\section{H. Broken time-reversal symmetry}

The case $\beta=2$ of broken time-reversal symmetry is less important for optical applications, but has been realized in microwave experiments [24-26] Theie is now no difference between $n=m$ and $n \neq m$ The matrices $U$ and $V$ have the same statistical distribution as for the case of preserved timereveisal symmetry Hence, by following the steps of Sec III A, we anive again at Eq (26), with spectral moments $B_{k}$ as defined in Eq (23) Theil joint distribution has now to be calculated fiom Eq (8) with $\beta=2$ This calculation is carried out in Appendix B The result is

$$
P\left(B_{1}, B_{2}\right)=\frac{2 \gamma N^{3} B_{1}^{2}}{B_{2}^{3}} \exp \left(-N B_{1}^{2} / B_{2}-2 \gamma N / B_{1}\right)
$$

The distibution of single-mode delay times $P\left(\phi^{\prime}\right)$ is given by Eq (28), with the function $P\left(B_{1}, B_{2}\right)$ We plot $P\left(\phi^{\prime}\right)$ in Fig 7, and compare it to the case of preserved time-reversal symmetiy The distilbution is rescaled by about a factor of 2 towatd laiget delay times when time-reversal symmetry is broken This can be understood from the fact that the relevant length scale, the localization length, is twice as large for bioken time-1eversal symmetry $(\xi=2 N L / s$, while $\xi$ $=N L / s$ for preserved tume-reversal symmetry)

\section{CONCLUSION}

We have presented a detailed theory, supported by numeical simulations, of a recently discovered [7] coherent backscatteing effect in the single-mode delay times of a wave reflected by a disordered waveguide This dynamic effect is special because it requires localization for its existence, in contrast to the static coherent backscatteing effect in the reflected intensity The dynamic effect can be under stood from the combination of the static effect and the latge 
fluctuations in the localized regime.

In the diffusive regime there is no dynamic coherent backscattering effect: The distribution of delay times is unaffected by the choice of the detection mode and the presence or absence of time-reversal symmetry. The effect also disappears when the absorption length is smaller than the localization length. In both situations the large fluctuations characteristic of the localized regime are suppressed.

Existing experiments on the delay-time distribution $[11,12]$ verified the diffusion theory $[13]$. The theory for the localized regime presented here awaits experimental verification.

\section{ACKNOWLEDGMENTS}

We thank P. W. Brouwer for valuable advice. This work was supported by the "Nederlandse organisatie voor Wetenschappelijk Onderzoek" (NWO) and by the "Stichting voor Fundamenteel Onderzoek der Materie"' (FOM).

\section{APPENDIX A: JOINT DISTRIBUTION OF $B_{1}$ AND $B_{2}$ FOR $\beta=1$}

We calculate the joint probability distribution function $P\left(B_{1}, B_{2}\right)$ of the spectral moments $B_{1}$ and $B_{2}$, defined in Eq. (23), which determine $P\left(I, \phi^{\prime}\right)$ from Eq. (26). We assume preserved time-reversal symmetry $(\beta=1)$. Since $B_{k}$ $=\Sigma_{l}\left|u_{l}\right|^{2} \mu_{l}^{-k}$, we have to average over the wave function amplitudes $u_{l}$, which are Gaussian complex numbers with zero mean and variance $1 / N$, and the rates $\mu_{t}$ which are distributed according to the Laguerre ensemble [Eq. (8)] with $\beta=1$. This Laguerre ensemble is represented as the eigenvalues of an $N \times N$ Hermitian matrix $W^{\dagger} W$, where $W$ is a complex symmetric matrix with the Gaussian distribution:

$$
P(W) \propto \exp \left[-\gamma(N+1) \operatorname{tr} W^{\dagger} W\right] .
$$

The calculation is performed neglecting corrections of order $1 / N$, so that we are allowed to replace $N+1$ by $N$. The measure is

$$
d W=\prod_{l<j} d \operatorname{Re} W_{i j} d \operatorname{Im} W_{t j} \prod_{l} d \operatorname{Re} W_{t \imath} d \operatorname{Im} W_{l l}
$$

\section{Characteristic function}

In the first step we express $P\left(B_{1}, B_{2}\right)$ by its characteristic function,

$$
\begin{gathered}
P\left(B_{1}, B_{2}\right)=\frac{1}{(2 \pi)^{2}} \int_{-\infty}^{\infty} d p \int_{-\infty}^{\infty} d q e^{i p B_{1}+\imath q B_{2}} \chi(p, q), \\
\chi(p, q)=\left\langle\prod_{l=1}^{N} \exp \left[-i\left|u_{l}\right|^{2}\left(\frac{p}{\mu_{l}}+\frac{q}{\mu_{l}^{2}}\right)\right]\right\rangle,
\end{gathered}
$$

and average over the $u_{l}$ 's:

$$
\begin{aligned}
\chi(p, q) & =\left\langle\prod_{l=1}^{N}\left(1+i \frac{p}{\mu_{l} N}+i \frac{q}{\mu_{l}^{2} N}\right)^{-1}\right\rangle \\
& =\left\langle\frac{\operatorname{det}\left(W^{\dagger} W\right)^{2}}{\operatorname{det}\left[\left(W^{\dagger} W\right)^{2}+i p\left(W^{\dagger} W\right) / N+i q / N\right]}\right\rangle
\end{aligned}
$$

We have expressed the product over eigenvalues as a ratio of determinants. We write the determinant in the denominator as an integral over a complex vector $\mathbf{z}$ :

$$
\begin{aligned}
\chi(p, q) \propto & \int d W \int d \mathbf{z} \exp \left[-\gamma N \operatorname{tr} W^{\dagger} W\right] \operatorname{det}\left(W^{\dagger} W\right)^{2} \\
& \times \exp \left\{-\mathbf{z}^{\dagger}\left[\left(W^{\dagger} W\right)^{2}+i p\left(W^{\dagger} W\right) / N+i q / N\right] \mathbf{z}\right\} .
\end{aligned}
$$

This integral converges because $W^{\dagger} W$ is positive definite.

\section{Parametrization of the matrix $W$}

Now we choose a parametrization of $W$ which facilitates a stepwise integration over its degrees of freedom. The distribution of $W$ is invariant under transformations $W \rightarrow U^{T} W U$, with any unitary matrix $U$. Hence we can choose a basis in which $\mathbf{z}$ points in direction 1 , and write $W$ in block form:

$$
W=\left(\begin{array}{ll}
a & \mathbf{x}^{T} \\
\mathbf{x} & X
\end{array}\right) .
$$

Here $a$ is a complex number. For any $(N-1)$-dimensional vector $\mathbf{x}$ we can use another unitary transformation on the $X$ block after which $\mathbf{x}$ points in direction 2 . Then $W$ is of the form

$$
W=\left(\begin{array}{lll}
a & x & 0^{T} \\
x & b & \mathbf{y}^{T} \\
0 & \mathbf{y} & Y
\end{array}\right),
$$

with the real number $x=|\mathbf{x}|$. In this parametrization

$$
\begin{gathered}
\left(W^{\dagger} W\right)_{11}=|a|^{2}+x^{2}, \\
{\left[\left(W^{\dagger} W\right)^{2}\right]_{11}=\left(|a|^{2}+x^{2}\right)^{2}+x^{2} y^{2}+x^{2}\left|a+b^{*}\right|^{2},} \\
\operatorname{det} W=\left[a\left(b-\mathbf{y}^{T} Y^{-1} \mathbf{y}\right)-x^{2}\right] \operatorname{det} Y, \\
\operatorname{tr} W^{\dagger} W=|a|^{2}+|b|^{2}+2 x^{2}+2 y^{2}+\operatorname{tr} Y, \\
d W=d^{2} a d^{2} b d \mathbf{x} d \mathbf{y} d Y,
\end{gathered}
$$

with $y=|\mathbf{y}|$. A suitable transformation on $Y$ allows one to replace the term $\mathbf{y}^{T} Y^{-1} \mathbf{y}$ by $y^{2}\left(Y^{-1}\right)_{11}$.

For this parametrization of $W$, the integrand in Eq. (A6) depends on the vectors $\mathbf{x}, \mathbf{y}$, and $\mathbf{z}$ only by their magnitudes $x, y$, and $z=|z|$. Hence we can replace $d \mathbf{x} \rightarrow x^{2 N-3} d x, d \mathbf{y}$ $\rightarrow y^{2 N-5} d y$, and $d \mathbf{z} \rightarrow z^{2 N-1} d z$. 


\section{Integration}

The integrand in Eq. (A6) involves $z, p$, and $q$ in the form

$$
\exp \left[-z^{2}\left(\left[\left(W^{\dagger} W\right)^{2}\right]_{11}+i p\left(W^{\dagger} W\right)_{11} / N+i q / N\right)\right]
$$

It is convenient to pass back to $P\left(B_{1}, B_{2}\right)$ by Eq. (A3), because integration over $p$ and $q$ gives delta functions:

$$
\begin{aligned}
& \delta\left[B_{1}-z^{2}\left(|a|^{2}+x^{2}\right) / N\right] \delta\left(B_{2}-z^{2} / N\right) \\
& \quad=\frac{1}{B_{2}} \delta\left(B_{1} / B_{2}-|a|^{2}-x^{2}\right) \delta\left(B_{2}-z^{2} / N\right) .
\end{aligned}
$$

Subsequent integration over $z$ results in

$$
\begin{aligned}
P\left(B_{1}, B_{2}\right) \propto & \int d^{2} a d^{2} b d x d y x^{2 N-3} y^{2 N-5} B_{2}^{N-2} \delta \\
& \times\left(B_{1} / B_{2}-|a|^{2}-x^{2}\right)\left[c_{0}\right. \\
& \left.\times\left|a b-x^{2}\right|^{4}+4 c_{2}|a|^{2} y^{4}\left|a b-x^{2}\right|^{2}+c_{4}|a|^{4} y^{8}\right] \\
& \times \exp \left[-N B_{2}\left(\frac{B_{1}^{2}}{B_{2}^{2}}+x^{2} y^{2}+x^{2}\left|a^{*}+b\right|^{2}\right)\right. \\
& \left.-2 \gamma N y^{2}\right]
\end{aligned}
$$

Here we omitted a term $\gamma N\left(|a|^{2}+|b|^{2}+2 x^{2}\right)$ in the exponent, because it is of order $1 / N$, as we shall see later. Furthermore, we denoted

$$
c_{m}=\frac{\left\langle|\operatorname{det} Y|^{4}\left|\left(Y^{-1}\right)_{11}\right|^{m}\right\rangle}{\left\langle|\operatorname{det} Y|^{4}\right\rangle} .
$$

These coefficients will be calculated later, with the results $c_{0}=1, c_{2}=2 \gamma$, and $c_{4}=4 \gamma^{2}$. Integration over $y$ yields for the terms proportional to $c_{m}$ the factors $\left(B_{2} x^{2}\right.$ $+2 \gamma)^{-m-N+2}$, which can be combined with the factor $\left(B_{2} x^{2}\right)^{N-2}$, giving, to order $1 / N$ [we anticipate $\gamma / B_{2} x^{2}$ $=O(1 / N)]$,

$\frac{\left(B_{2} x^{2}\right)^{N-2}}{\left(B_{2} x^{2}+2 \gamma\right)^{N-2+m}} \rightarrow\left(B_{2} x^{2}\right)^{-m} \exp \left(-\frac{2 \gamma N}{B_{2} x^{2}}\right)$.

We introduce a new integration variable by $b^{\prime}=b+a^{*}$. So far $P\left(B_{1}, B_{2}\right)$ is reduced to the form

$$
\begin{aligned}
P\left(B_{1}, B_{2}\right) \propto & \int d^{2} a d^{2} b^{\prime} d x x \delta\left(B_{1} / B_{2}-|a|^{2}-x^{2}\right) \\
& \times\left(\left|a b^{\prime}-\frac{B_{1}}{B_{2}}\right|^{4}+\frac{4 c_{2}|a|^{2}}{B_{2}^{2} x^{4}}\right. \\
& \left.\times\left|a b^{\prime}-\frac{B_{1}}{B_{2}}\right|^{2}+\frac{c_{4}|a|^{4}}{B_{2}^{4} x^{8}}\right) \\
& \times \exp \left(-\frac{2 \gamma N}{x^{2} B_{2}}-N \frac{B_{1}^{2}}{B_{2}}-N B_{2} x^{2}\left|b^{\prime}\right|^{2}\right) .
\end{aligned}
$$

Let us now convince ourselves with this expression that we were justified in omitting the term $\gamma N\left(|a|^{2}+|b|^{2}+2 x^{2}\right)$ in Eq. (A11) and in using Eq. (A13). Indeed, the various quantities scale as $B_{1} \simeq \gamma N, \quad B_{2} \simeq \gamma^{2} N^{3}$, and $|a|^{2} \simeq|b|^{2} \simeq x^{2}$ $\approx 1 / \gamma N^{2}$, because any $\gamma$ and $N$ dependence disappears if one passes to appropriately rescaled quantities $B_{1} / \gamma N$, etc. The terms omitted are therefore of order $1 / N$.

The remaining integrations in Eq. (A14) are readily performed, with the final result Eq. (27). The distribution of $B_{1}$, to order $1 / N$, is

$$
P\left(B_{1}\right)=\frac{\gamma N}{B_{1}^{3}}\left(B_{1}+2 \gamma N\right) \exp \left(-\frac{2 \gamma N}{B_{1}}\right)
$$

The spectral moment $B_{1}$ appeared before in a different physical context in Ref. [20], but only a heuristic approximation was given in that paper. Equation (A15) solves this random-matrix problem precisely.

For completeness we also give the distribution of the other spectral moment $B_{2}$ (rescaled as $\widetilde{B}_{2}=B_{2} \gamma^{-2} N^{-3}$ ) in terms of Meijer $G$ functions:

$$
\begin{aligned}
P\left(\widetilde{B}_{2}\right)= & \frac{1}{64 \widetilde{B}_{2}^{5 / 2} \pi^{1 / 2}}\left[14 \pi \widetilde{B}_{2}-16 G_{3,0}^{0,3}\left(\widetilde{B}_{2} \mid-\frac{1}{2}, 0, \frac{3}{2}\right)\right. \\
& +20 G_{3,0}^{0,3}\left(\widetilde{B}_{2} \mid-\frac{1}{2}, \frac{1}{2}, 1\right)+22 G_{3,0}^{0,3}\left(\widetilde{B}_{2} \mid \frac{1}{2}, \frac{1}{2}, 1\right) \\
& +8 G_{3,0}^{0,3}\left(\widetilde{B}_{2} \mid \frac{1}{2}, 1, \frac{3}{2}\right)+4 G_{3,0}^{0,3}\left(\widetilde{B}_{2} \mid \frac{1}{2}, \frac{3}{2}, 2\right) \\
& -8 G_{4,1}^{1,3}\left(\widetilde{B}_{2} \mid \begin{array}{c}
-\frac{1}{2}, 0, \frac{3}{2}, 2 \\
1
\end{array}\right)-16 G_{4,1}^{1,3}\left(\widetilde{B}_{2} \mid \begin{array}{c}
0, \frac{1}{2}, \frac{3}{2}, 2 \\
1
\end{array}\right) \\
& \left.+3 G_{4,1}^{0,4}\left(\widetilde{B}_{2} \mid \begin{array}{c}
\frac{1}{2}, \frac{1}{2}, \frac{3}{2}, \frac{3}{2} \\
0
\end{array}\right)\right] .
\end{aligned}
$$

\section{Coefficients}

Now we calculate the coefficients $c_{2}$ and $c_{4}$ defined in Eq. (A12). It is convenient to resize the matrix $Y$ to dimension $N$ (instead of $N-2$ ), and to set $\gamma N=1$ momentarily. We again use a block decomposition,

$$
Y=\left(\begin{array}{cc}
a & \mathbf{w}^{T} \\
\mathbf{w} & Z
\end{array}\right)
$$

and employ the identities

$$
\begin{aligned}
& \operatorname{det} Y=\left(a-\mathbf{w}^{T} Z^{-1} \mathbf{w}\right) \operatorname{det} Z, \\
& \left(Y^{-1}\right)_{11}=\left(a-\mathbf{w}^{T} Z^{-1} \mathbf{w}\right)^{-1} .
\end{aligned}
$$

Hence

$$
c_{4}=\frac{\left\langle|\operatorname{det} Z|^{4}\right\rangle}{\left\langle|\operatorname{det} Y|^{4}\right\rangle}=\frac{4}{(N+1)(N+3)},
$$

where we used Selberg's integral [27] for 


$$
\left\langle|\operatorname{det} Y|^{4}\right\rangle=\frac{1}{6} \frac{\Gamma(N+4) \Gamma(N+2)}{2^{2 N}}
$$

In order to evaluate

$$
c_{2}=\frac{\left\langle|\operatorname{det} Z|^{4}\left(|a|^{2}+\left|w^{T} Z^{-1} w\right|^{2}\right)\right\rangle}{\left\langle|\operatorname{det} Y|^{4}\right\rangle},
$$

it is again profitable to use unitary invariance and turn $\mathbf{w}$ in direction 1:

$$
\left|\mathbf{w}^{T} Z^{-1} \mathbf{w}\right|^{2}=w^{4}\left|\left(Z^{-1}\right)_{11}\right|^{2}
$$

From $\left\langle w^{4}\right\rangle=\frac{1}{4} N(N+1)$ and $\left\langle|a|^{2}\right\rangle=1$ we then obtain the recursion relation

$$
c_{2}(N)=\frac{4}{(N+1)(N+3)}+\frac{N}{N+3} c_{2}(N-1),
$$

which is solved by

$$
c_{2}(N)=\frac{2}{N+1}
$$

In order to reintroduce $\gamma$ we have to multiply $c_{m}$ by $(\gamma N)^{m / 2}$, and obtain, to order $1 / N$,

$$
c_{2}=2 \gamma, \quad c_{4}=4 \gamma^{2}
$$

as advertised above.

\section{APPENDIX B: JOINT DISTRIBUTION OF $B_{1}$ AND $B_{2}$ FOR $\beta=2$}

For broken time-reversal symmetry, the distributions of $B_{1}$ and $B_{2}$ have to be calculated from the Laguerre ensemble [Eq. (8)] with $\beta=2$. Similarly as for preserved time-reversal symmetry, this ensemble can be obtained from the eigenvalues of a matrix $W^{\dagger} W$. The matrix $W$ is once more complex, but no longer symmetric (it is also not Hermitian). It has a Gaussian distribution

$$
P(W) \propto \exp \left(-2 \gamma N \operatorname{tr} W^{\dagger} W\right),
$$

with measure

$$
d W=\prod_{i, j} d \operatorname{Re} W_{i j} d \operatorname{Im} W_{i j}
$$

It is instructive to calculate $P\left(B_{1}\right)$ first, because it will be instrumental in the calculation of $P\left(B_{1}, B_{2}\right)$. After averaging over the $u_{i}$ 's, the characteristic function takes the form

$$
\chi(p)=\left\langle\exp \left(-i p B_{1}\right)\right\rangle=\left\langle\frac{\operatorname{det} W^{\dagger} W}{\operatorname{det}\left(W^{\dagger} W+i p / N\right)}\right\rangle
$$

We express the determinant in the denominator as an integral over a complex vector $\mathbf{z}$. Due to the invariance $W \rightarrow U W V$ of $P(W)$ for arbitrary unitary matrices $U$ and $V$, we can turn $\mathbf{z}$ in direction 1 , and write

$$
W=\left(\begin{array}{lll}
a & x^{\prime} & 0^{T} \\
x & & \\
& & Y \\
0 & &
\end{array}\right)
$$

Then

$$
\begin{aligned}
P\left(B_{1}\right) \propto & \int d p d z z^{2 N-1} d^{2} a d x x^{2 N-3} d x^{\prime} x^{\prime 2 N-3} \\
& \times\left(|a|^{2}+d_{2} x^{2} x^{\prime 2}\right) \exp \left[-\left(z^{2}+2 \gamma N\right)\left(|a|^{2}+x^{2}\right)\right] \\
& \times \exp \left[i p\left(B_{1}-z^{2} / N\right)-2 \gamma N x^{\prime 2}\right]
\end{aligned}
$$

Selberg's integral [27] gives

$$
d_{2} \equiv \frac{\left\langle|\operatorname{det} Y|^{2}\left|\left(Y^{-1}\right)_{11}\right|^{2}\right\rangle}{\left\langle|\operatorname{det} Y|^{2}\right\rangle}=\frac{2 \gamma N}{N-1} .
$$

The integration over $p$ gives $\delta\left(z^{2}-N B_{1}\right)$, and allows one to eliminate $z$. The integration over $x^{\prime}$ amounts to replacing $x^{\prime 2}=(N-1) / 2 \gamma N=d_{2}^{-1}$. The final integrations are most easily carried out by concatenating $a$ to $\mathbf{x}$, giving an $N$-dimensional vector $\mathbf{y}$. Then

$$
\begin{aligned}
P\left(B_{1}\right) \propto & \int d y y^{2 N+1} B_{1}^{N-1} \\
& \times \exp \left[-N\left(B_{1}+2 \gamma\right) y^{2}\right] \\
\propto & B_{1}^{N-1}\left(B_{1}+2 \gamma\right)^{-N-1}
\end{aligned}
$$

which to order $1 / N$ becomes

$$
P\left(B_{1}\right)=\frac{2 \gamma N}{B_{1}^{2}} \exp \left(-2 \gamma N / B_{1}\right) .
$$

The first steps in the calculation of the joint distribution function of $B_{1}$ and $B_{2}$ are identical to what was done in Appendix $\mathrm{A}$, and result in the characteristic function $\chi(p, q)$ in the form of Eq. (A6), but with $\gamma$ replaced by $2 \gamma$. Due to the unitary invariance of the $W$ ensemble we can write

$$
W=\left(\begin{array}{cccc}
a & x^{\prime} & 0 & 0^{T} \\
x & b & y^{\prime} & 0^{T} \\
0 & y & & \\
& & & Y \\
0 & 0 & &
\end{array}\right)
$$

One now integrates over $p$ and $q$ and obtains delta functions as in Eq. (A10). This is followed by integration over $z$. The calculation is then much simplified by recognizing that one can rescale the remaining integration variables in such a way (namely, by introducing $a^{2}=\widetilde{a}^{2} B_{1} / B_{2}, x^{2}=\widetilde{x}^{2} B_{1} / B_{2}, y^{\prime 2}$ $=\tilde{y}^{\prime 2} x^{-2} B_{2}^{-1}$ ) that

$$
P\left(B_{1}, B_{2}\right)=B_{2}^{-3} \exp \left(-N B_{1}^{2} / B_{2}\right) f\left(B_{1}\right) .
$$


It is not necessary here to give $f\left(B_{1}\right)$ as a lengthy multidimensional integral, since its functional form is easily recovered from the relation

$$
P\left(B_{1}\right)=\int d B_{2} P\left(B_{1}, B_{2}\right)=N^{-2} B_{1}^{-4} f\left(B_{1}\right) .
$$

We compare this with Eq. (B8), and arrive at Eq. (41). The distribution of $B_{2}$ has the closed-form expression

$$
P\left(B_{2}\right)=\gamma^{-2} N^{-3} G_{3,0}^{0,3}\left(\gamma^{-2} N^{-3} B_{2} \mid-\frac{1}{2},-1,-2\right) .
$$

\section{APPENDIX C: JOINT DISTRIBUTION OF $C_{0}$ AND $C_{1}$}

We seek the joint distributions of the spectral moments $C_{0}$ and $C_{1}$, which determine $\phi^{\prime}$ and $I$ for $\beta=1$ and $n=m$ via Eq. (29). We start with the characteristic function

$$
\chi\left(p_{0}, p_{1}\right)=\left\langle\exp \left[i \operatorname{Re}\left(p_{0} C_{0}+p_{1} C_{1}\right)\right]\right\rangle,
$$

where $p_{0}$ and $p_{1}$ are complex numbers, as are the quantities $C_{0}$ and $C_{1}$ themselves. Since $C_{k}=\Sigma_{\imath} u_{l}^{2} \tau_{1}^{k}$, we have to average over the $\tau_{i}$ 's and the $u_{i}$ 's. Averaging over the $u_{i}$ 's first, we obtain

$$
\chi\left(p_{0}, p_{1}\right)=\left\langle\prod_{l}\left(1+\frac{\left|p_{1} \tau_{\imath}+p_{0}\right|^{2}}{N^{2}}\right)^{-1 / 2}\right\rangle .
$$

We again regard the rates $\mu_{t}=\tau_{t}^{-1}$ as the eigenvalues of a matrix product $Y Y^{\dagger}$, where $Y$ will be specified below. Then the product of square roots can be written as a ratio of determinants:

$$
\begin{aligned}
\prod_{t}\left(1+\frac{\left|p_{1} \tau_{l}+p_{0}\right|^{2}}{N^{2}}\right)^{-1 / 2} \\
=\operatorname{det} Y Y^{T} \operatorname{det}\left[\left(Y Y^{T}\right)^{2} \frac{N^{2}+\left|p_{0}\right|^{2}}{N^{2}}\right. \\
\left.+2 \frac{\operatorname{Re} p_{0} p_{1}^{*}}{N^{2}} Y Y^{T}+\frac{\left|p_{1}\right|^{2}}{N^{2}}\right]^{-1 / 2} .
\end{aligned}
$$

We will express the determinant in the denominator as a Gaussian integral over a real $N$-dimensional vector $\mathbf{z}$. Hence it is convenient to choose $Y$ real as well, so that one can use orthogonal invariance in order to turn $\mathbf{z}$ in direction 1. Moreover, there is a representation of $Y$ which allows one to incorporate the determinant in the numerator into the probability measure: We take $Y$ as a rectangular $N \times(N+3)$ matrix with random Gaussian variables, distributed according to

$$
P(Y) \propto \exp \left(-\gamma N \operatorname{tr} Y Y^{T}\right)
$$

The corresponding distribution of the eigenvalues $\mu_{l}$ of $Y Y^{T}$ is given in Ref. [23], and differs from the Laguerre ensemble [Eq. (8)] by the additional factor $\Pi_{i} \mu_{i}=\operatorname{det} Y Y^{T}$. In this representation,

$$
\begin{aligned}
\chi^{\propto} \int d z z^{N-1}\left\langle\exp \left\{-z^{2}\left(1+\left|p_{0}\right|^{2} / N^{2}\right)\left[\left(Y Y^{T}\right)^{2}\right]_{11}\right\}\right. \\
\left.\times \exp \left[\frac{2 \operatorname{Re} p_{0} p_{1}^{*}}{N^{2}}\left[Y Y^{T}\right]_{11}+\frac{\left|p_{1}\right|^{2}}{N^{2}}\right]\right),
\end{aligned}
$$

where the average is now over $Y$. Inverse Fourier transformation with respect to $p_{0}$ and $p_{1}$ results in

$$
\begin{aligned}
P\left(C_{0}, C_{1}\right) \propto & \left\langle\int d z z^{N-5} \frac{\exp \left[-z^{2}\left[\left(Y Y^{T}\right)^{2}\right]_{11}\right]}{\left[\left(Y Y^{T}\right)^{2}\right]_{11}-\left(\left[Y Y^{T}\right]_{11}\right)^{2}}\right. \\
& \times \exp \left[-\frac{\left|C_{1}\right|^{2} N^{2}}{4 z^{2}}-\frac{N^{2}}{4 z^{2}}\right. \\
& \left.\left.\times \frac{\left|C_{0}-\left[Y Y^{T}\right]_{11} C_{1}\right|^{2}}{\left[\left(Y Y^{T}\right)^{2}\right]_{11}-\left(\left[Y Y^{T}\right]_{11}\right)^{2}}\right]\right) .
\end{aligned}
$$

The orthogonal invariance of $Y Y^{T}$ allows us to parametrize $Y$ as

$$
Y=\left(\begin{array}{lll}
a & v & 0^{T} \\
w & b & \\
0 & y & Z \\
0 & 0 &
\end{array}\right)
$$

with real numbers $v>0, w>0, y>0, a$, and $b$, and an $[(N-1) \times(N+1)]$-dimensional matrix $Z$. It is good to see that $Z$ drops out of the calculation, because it does not appear in

$$
\begin{gathered}
{\left[Y Y^{T}\right]_{11}=a^{2}+v^{2}} \\
{\left[\left(Y Y^{T}\right)^{2}\right]_{11}=\left(a^{2}+v^{2}\right)^{2}+(a w+v b)^{2}+v^{2} y^{2}}
\end{gathered}
$$

We replace $b=b^{\prime}-a w / v$, and introduce $z^{\prime}=z y v$. The integral over $z^{\prime}$ can be written in the saddle-point form $\int d z^{\prime} z^{\prime N} e^{-z^{\prime 2}} f\left(z^{\prime}\right) \propto f(\sqrt{N / 2})$ for large $N$. The resulting expression varies with respect to the remaining variables on the scales

$$
N^{3} a^{2} \simeq N^{2} b^{\prime 2} \simeq N^{2} v^{2} \simeq N y^{2} \simeq w^{2}=O\left(\gamma^{-1}\right)
$$

We use the given orders of magnitude to eliminate terms of order $N^{-1}$, but keep the residual correlations $\operatorname{Re} C_{0} C_{1}^{*} / \gamma N$ $=O\left(N^{-1 / 2}\right)$. The joint distribution function of $C_{0}$ and $C_{1}$ is then 


$$
\begin{aligned}
P\left(C_{0}, C_{1}\right) \propto & \int d a d b^{\prime} d v v^{3} d w w^{N-2} d y y \exp \left[-\gamma N y^{2}\right] \\
& \times \exp \left[-\gamma N w^{2}\left(1+\frac{a^{2}}{v^{2}}\right)-\frac{N}{2 y^{2}}\left(v^{2}+y^{2}+b^{\prime 2}\right)\right] \\
& \times \exp \left[N v^{2} \operatorname{Re} C_{0} C_{1}^{*}-\frac{N v^{2} y^{2}\left|C_{1}\right|^{2}}{2}-\frac{N\left|C_{0}\right|^{2}}{2}\right]
\end{aligned}
$$

Now we can integrate over $a, b^{\prime}, w$, and $v$, and arrive at

$$
P\left(C_{0}, C_{1}\right) \propto \int d y \frac{\exp \left[-\gamma N y^{2}-N\left|C_{0}\right|^{2} / 2\right]}{\left(y^{-2}+y^{2}\left|C_{1}\right|^{2}+2 \operatorname{Re} C_{0} C_{1}^{*}\right)^{5 / 2}}
$$

The final result $[\mathrm{Eq}(30)]$ is obtained by substituting $s$ $=\gamma N y^{2}$

\section{APPENDIX D: DISTRIBUTION OF $A_{1}$ FOR $\beta=1$}

In the large- $N$ limit the joint distribution function $P\left(A_{0}, A_{1}\right)=P\left(A_{0}\right) P\left(A_{1}\right)$ factorizes, as explained in Sec III $\mathrm{E}$ The distribution of $A_{0}$ is given in Eq (37) It remains to calculate the distribution of $A_{1}=\Sigma_{t} \tau_{t} u_{t} v_{t}$ The $u_{l}$ 's and $v_{i}$ 's are independent Gaussian random numbers Averaging over them, we obtain the characteristic function

$$
\begin{aligned}
\chi(p) & =\left\langle\exp \left[\iota \operatorname{Re}\left(p A_{1}\right)\right]\right\rangle=\left\langle\prod_{t}\left(1+\frac{\left|p \tau_{1}\right|^{2}}{4 N^{2}}\right)^{-1}\right\rangle \\
& =\left\langle\frac{\operatorname{det}\left(W^{\dagger} W\right)^{2}}{\operatorname{det}\left[\left(W^{\dagger} W\right)^{2}+|p|^{2} / 4 N\right]}\right\rangle,
\end{aligned}
$$

where $p$ is a complex number The Laguerre ensemble is again represented as the eigenvalues of the matrix product $W^{\dagger} W$, where $W$ is the complex symmetric matrix with distribution (A1) Following the route of Appendix A we represent the determinant in the denominator by a Gaussian in tegral over a complex vector $\mathbf{z}$, and choose a basis in which $W 1$ s of the form of $\mathrm{Eq}$ (A8) The characteistic function is then obtained as the following multidimensional integral

$$
\begin{aligned}
\chi(p)= & \int d \mathbf{x} d \mathbf{y} d \mathbf{z} d^{2} a d^{2} b d Y \times|\operatorname{det} Y|^{4} \mid a\left[b-y^{2}\left(Y^{-1}\right)_{11}\right]^{2} \\
& -\left.x^{2}\right|^{4} \exp \left(-\frac{|z p|^{2}}{4 N^{2}}\right) \exp \left[-z^{2}\left(\left(|a|^{2}+x^{2}\right)^{2}+x^{2} \mid a\right.\right. \\
& \left.\left.+\left.b^{*}\right|^{2}+x^{2} y^{2}\right)\right] \exp \left[-\gamma N\left(|a|^{2}+|b|^{2}\right.\right. \\
& \left.\left.+2 x^{2}+2 y^{2}+\operatorname{tr} Y^{\dagger} Y\right)\right]
\end{aligned}
$$

Let us briefly desciıbe in which order the integrations are performed most conveniently Fourier transformation with respect to $p$ converts the characteristic function back into the distribution function $P\left(A_{1}\right)$ This step gives rise to a factor $z^{-2} \exp \left(-\left|A_{1}\right|^{2} N^{2} z^{-2}\right)$ We can also integrate over $\mathbf{y}$, which results in a factor $\exp \left[-2 \gamma N /(x z)^{2}\right]$ We introduce new var1ables by the substitutions $b=\tilde{b}-a^{*}, \mathbf{x}=\mathbf{v} / z$, and $a=a^{\prime} / z$ After these transformations one succeeds in integrating over $b^{\prime}, \mathbf{z}$, and $a^{\prime}$ The remaining integral over $v=|\mathbf{v}|$ is of the form

$$
\begin{aligned}
& P\left(A_{1}\right) \propto\left|A_{1}\right|^{-5} \int d v v^{-5} e^{-2 / v} \\
& \times\left[\pi\left[8\left|A_{1}\right|^{2}\left(2+4 v+v^{2}\right)+3 v^{2}\left(16+16 v+3 v^{2}\right)\right]\right. \\
&-\frac{2\left|A_{1}\right| v}{\left(\left|A_{1}\right|^{2}+v^{2}\right)^{4}}\left[\left|A_{1}\right|^{4} v^{4}\left(288+304 v-25 v^{2}\right)\right. \\
&+\left|A_{1}\right|^{6} v^{2}\left(192+176 v-17 v^{2}\right) \\
&+8\left|A_{1}\right|^{8}\left(6+4 v-v^{2}\right)+3 v^{8}\left(16+16 v+3 v^{2}\right) \\
&\left.+\left|A_{1}\right|^{2} v^{6}\left(192+208 v+41 v^{2}\right)\right] \\
&-\left[16\left|A_{1}\right|^{2}\left(2+4 v+v^{2}\right)+6 v^{2}\left(16+16 v+3 v^{2}\right)\right] \\
&\left.\times \arctan \left(\frac{v}{\left|A_{1}\right|}\right)\right]
\end{aligned}
$$

The more compact form $[\mathrm{Eq}(38)]$ is the result of the replacement $v=2 / s$, followed by a number of partial integrations
[1] A Ishımaru, Wave Propagatıon and Scattering in Random Media (Academic, New York, 1978)

[2] P Sheng, Scattering and Localization of Classical Waves th Random Media (World Scientıfic, Singapore, 1990)

[3] R Berkovits and S Feng, Phys Rep 238, 135 (1994)

[4] M P van Albada and A Lagendik, Phys Rev Lett 55, 2692 (1985)

[5] P -E Wolf and G Maret, Phys Rev Letl 55, 2696 (1985)

[6] S John, Phys Today 44(5), 32 (1991)

[7] $\mathrm{H}$ Schomerus, KJH van Bemmel, and $\mathrm{CWJ}$ Beenakker, Europhys Lett 52, 512 (2000)

[8] E P Wigner, Phys Rev 98, 145 (1955)
[9] F T Smith, Phys Rev 118, 349 (1960)

[10] Y V Fyodorov and H -J Sommers, J Math Phys 38, 1918 (1997)

[11] AZ Genack, P Sebbah, M Stoytchev, and B A van Tiggelen, Phys Rev Lett 82, 715 (1999)

[12] A Lagendijk, J Gómez Rivas, A Imhof, F J P Schuurmans, and R Sprik, in Photonic Crystals and Light Localization, NATO Advanced Study Institute Senes, edited by C M Soukoulis (Kluwer, Doidrecht, in press)

[13] B A van Tiggelen P Sebbah, M Stoytchev, and A Z Genack, Phys Rev E 59, 7166 (1999)

[14] CW J Beenakker and PW Brouwer, e pint cond-mat $/ 9908325$ 
[15] A M Jayannavar, G V Vijayagovindan, and $\mathrm{N}$ Kumar, $\mathrm{Z}$ Phys B Condens Matter 75, 77 (1989)

[16] J Heinrichs, J Phys Condens Matter 2, 1559 (1990)

[17] A Comtet and C Texier, J Phys A 30, 8017 (1997)

[18] P A Mello and A D Stone, Phys Rev B 44, 3559 (1991)

[19] C W J Beenakker, $\mathrm{KJ} \mathrm{H}$ van Bemmel, and P W Brouwer, Phys Rev E 60, R6313 (1999)

[20] C W J Beenakker, J C J Paasschens, and P W Brouwer, Phys Rev Lett 76, 1368 (1996)

[21] H U Baranger, D P DiVincenzo, R A Jalabert, and A D Stone, Phys Rev B 44, 10637 (1991)
[22] C W J Beenakker, Rev Mod Phys 69, 731 (1997)

[23] A Edelman, Linear Algebr Appl 159, 55 (1991)

[24] F Erbacher, R Lenke, and G Maret, Europhys Lett 21, 551 (1993)

[25] H Alt, H -D Graf, H L Harney, R Hofferbert, H Lengeler, A Richter, P Schardt, and HA Weidenmuller, Phys Rev Lett 74, 62 (1995)

[26] U Stoffregen, J Steın, H -J Stockmann, M Kuś, and F Haake, Phys Rev Lett 74, 2666 (1995)

[27] M Mehta, Random Matrces (Academic, New York, 1990) 